\title{
Einige chemische Gesichtspunkte zur Wasserozonisierung
}

\section{VON WERNER STUMM}

Eidg. Anstalt für Wasserversorgung, Abwasserreinigung und Gewässerschutz an der Eidg. Technischen Hochschule Zürich, Mitteilung Nr. 107

Manuskript eingegangen am 6. August 1956

\section{EINLEITUNG}

Die Anwendung von Ozon zur Desinfektion von Trinkwasser ist älter als die Chlorierung. Trotzdem weiss man heute über die theoretischen Grundlagen der Wasserozonisierung und über die bakteriziden Eigenschaften des Ozons recht wenig. Dies hat seine Ursache vermutlich darin, dass die chemischen und physikalisch-chemischen Eigenschaften wässeriger Ozonlösungen noch nicht genügend untersucht sind.

Erst die Kenntnis der chemischen Reaktionseigenschaften eines Entkeimungsmittels liefert die notwendigen Grundlagen für ein umfassenderes Verständnis der Vorgänge bei der Keimtötung. So erwies sich zum Beispiel die Chemie der Chlorierung (FAIR und Mitarbeiter [ []$^{I}$ )) als wichtige Grundlage für die richtige Anwendung des Chlors in der Wasserpraxis. Es zeigte sich bekanntlich, dass bei der Zugabe von Chlor je nach $\mathrm{pH}$ und nach dem Verunreinigungsgrad des Wassers verschiedene Verbindungen mit verschiedenen bakteriziden Eigenschaften entstehen. Neben elementarem Chlor treten unterchlorige Säure (HOCl), Hypochlorition $\left(\mathrm{OCl}^{-}\right)$, Chloramine $\left(\mathrm{NH}_{2} \mathrm{Cl}, \mathrm{NHCl}_{2}, \mathrm{NCl}_{3}\right)$ und komplexe organische Chloramine auf. Die mannigfaltigen Reaktionsmöglichkeiten erschweren seine sachgemässe Anwendung.

Beim Ozon scheinen auf den ersten Blick die Verhältnisse bedeutend einfacher zu sein. So kann zum Beispiel Ozon in wässeriger Lösung, im Gegensatz zu Chlor, keine Protolysenreaktion (Säure-Base-Reaktion) eingehen. Trotzdem sind wir durch unsere Versuche zur Auffassung gekommen,

1) Die Ziffern in eckigen Klammern verweisen auf das Literaturverzeichnis, Seite 207. 
dass die Chemie wässeriger Ozonlösungen sehr kompliziert ist. Wenn wir auch nicht in der Lage sind, unsere Resultate vollumfänglich zu interpretieren, so möchten wir trotzdem über einige Beobachtungen berichten, die uns für die Wasserozonisierung wesentlich zu sein scheinen²).

\section{EINIGE CHEMISCHE}

\section{UND PHYSIKALISCH-CHEMISCHE EIGENSCHAFTEN WÄSSERIGER OZONLÖSUNGEN}

Ozon zerfällt in wässeriger Lösung erheblich schneller als im Gaszustand. Der Ozonzerfall erfolgt in wässeriger Lösung nach einer Reaktion erster Ordnung [2]. Die Zerfallskinetik weist darauf hin, dass eine Kettenreaktion unter Entstehung verschiedener $Z$ wischenprodụkte vor sich geht. Als mögliche Zwischenprodukte seien erwähnt: $\mathrm{HO}_{3}{ }^{+}, \mathrm{HO}_{2}, \mathrm{HO}, \mathrm{O}_{2}^{-}[2]$, [3], [4]. Diese Partikel sind kurzlebig und haben alle oxydierende Wirkung. Analoge Zerfallsprodukte sind bei der Zersetzung von Wasserstoffperoxyd festgestellt worden. Der Zerfall von Ozon in wässeriger Lösung wird durch zahlreiche Stoffe katalysiert. So ist zum Beispiel die Zerfallsreaktion in starkem Masse vom $\mathrm{pH}$ abhängig. Die Zerfallsgeschwindigkeit steigt mit zunehmender Hydroxylionenkonzentration stark an [2]. Der Zerfall wird auch durch gewisse Metalle und Metallionen, durch Wasserstoffperoxyd [4] und durch zahlreiche organische Substanzen (Fermente, Zucker usw.) in sehr starkem Masse katalysiert. Diese Zerfallsbeeinflussung durch gewisse organische Stoffe ist vergleichbar mit der Zerfallskatalyse von Wasserstoffperoxyd (zum Beispiel zerstört I Molekül Katalase innerhalb I Sekunde 2000 Moleküle $\mathrm{H}_{2} \mathrm{O}_{2}$ ). Natürliche Wässer sind in der Regel nicht ozonzehrungsfrei. Organische (evtl. auch anorganische) Verbindungen, die im Wasser enthalten sind, können durch Ozon oxydiert werden, wobei zahlreiche organische Oxydationsprodukte, zum Beispiel Ozonide, entstehen können. In wässerigen Ozonlösungen können also neben molekularem Ozon zahlreiche weitere oxydierend wirkende Substanzen (radikalartige Zerfallsprodukte und durch Ozon aufoxydierte organische Bestandteile, wie Ozonide und dergleichen), von denen man annehmen muss, dass sie ebenfalls bakterizide Eigenschaften aufweisen, vorhanden sein. In qualitativen Versuchen konnten wir feststellen, dass die Zerfallsprodukte von

${ }^{2}$ ) Wir sind den Herren Dr. K. Wuhrmann und Dr. F. Sulzer für wertvolle Anregungen zu grossem Dank verpflichtet. 
Ozon ebenfalls bakterizid sind, was auch durch Beobachtungen von WUHRMANN Und MEYRATH [ 5 ] bestätigt wird. Dies ist möglicherweise einer der Gründe für die sehr widerspruchsvollen Angaben in der Literatur über die bakterizide Wirkung des Ozons.

Eine andere Ursache der schlechten Übereinstimmung der Resultate verschiedener Autoren liegt in der Ozonbestimmungsmethode. Das häufig angewandte jodometrische Verfahren zur Bestimmung von Ozon in wässeriger Lösung ermittelt nicht nur den Ozongehalt, sondern die Summe aller oxydierend wirkenden Verbindungen (Ozon, Ozonzerfallsprodukte und aufoxydierte organische Verbindungen). Die an und für sich spezifisch auf Ozon ansprechende spektrophotometrische Absorptionsmessung von Ozon im UV. (Ozonlösungen absorbieren bei $260 \mathrm{~m} \mu$ ) ist leider in wässeriger Lösung wenig empfindlich. ALDER und HILL [3] haben darauf hingewiesen, dass wesentlich andere Zerfallsgeschwindigkeiten erhalten werden, je nachdem der Ozongehalt spektrophotometrisch oder jodometrisch ermittelt wird. Sie konnten überdies zeigen, dass die spektrophotometrisch bestimmte Zerfallsgeschwindigkeit stark von der Hydroxylionenkonzentration abhängig ist, während die jodometrisch erhaltenen Resultate eine weitgehende Unabhängigkeit der Zerfallskonstante vom pH aufweisen. Diese Beobachtung, welche mit unseren Versuchsergebnissen übereinstimmt, ist ein zusätzliches Indiz für die Unspezifität der jodometrischen Methode. Der Fehler der jodometrischen Ozonbestimmung scheint in nichtalkalischen, mit ozonzehrungsfreiem Wasser frisch hergestellten Ozon, lösungen gering, in ozonisierten natürlichen Wässern aber erheblich zu sein.

Die von ZEHENDER und STUMM [6] ausgearbeitete Analysenmethode für Ozon in wässeriger Lösung ist sehr empfindlich, recht spezifisch und praktisch einfach. Sie beruht auf der durch Ozon bewirkten Oxydation von zweiwertigem Mangan in saurer Lösung zu $\mathrm{MnO}_{2}$. Das gebildete $\mathrm{MnO}_{2}$ lässt man nach einer kurzen Wartefrist mit o-Tolidin reagieren, so dass die Ozonkonzentration mittels einer Farbreaktion photometrisch oder mit Hilfe von Vergleichslösungen ermittelt werden kann. Der Vorteil dieser Methode besteht vor allem darin, dass durch das System $\mathrm{Mn}^{+2} / \mathrm{MnO}_{2}$ peroxydähnliche Verbindungen (Ozonzerfallsprodukte und durch Ozon aufoxydierte organische Verbindungen) katalytisch vor der Farbstoffbildung zerstört werden. Die Selbstzersetzung von $\mathrm{H}_{2} \mathrm{O}_{2}$ und ähnlichen Verbindungen, wobei Wasser und Sauerstoff entstehen, wird bekanntlich [7] durch Redoxsysteme mit Potentialwerten, die zwischen den Reduktionsund Oxydationspotentialen von Peroxyd liegen, also zum Beispiel durch das System $\mathrm{Mn}^{+2} / \mathrm{MnO}_{2}$ stark katalysiert. (In sauren Lösungen beträgt 
der Mittelwert der genannten Potentialspanne I,23 V, während das Redoxpaar $\mathrm{Mn}^{+2} / \mathrm{MnO}_{2}$ ein Normalpotential von I,28 V aufzeigt.) Auch die organischen Ozonide, die als organische Peroxyde [8] aufzufassen sind, unterliegen unter diesen Bedingungen der Selbstzersetzung.

\section{OZONISIERUNG VON WASSER}

Wir haben in Laborversuchen verschiedene Wässer kontinuierlich und unter gleichen Bedingungen mit ozonhaltiger Luft $\left(0,3-3 \mathrm{mg} \mathrm{O}_{3} / \mathrm{I}\right)$ begast und die zeitliche Veränderung der Konzentration von Ozon in Wasser graphisch aufgetragen. Man erhält dabei Kurven, mit deren Hilfe eine

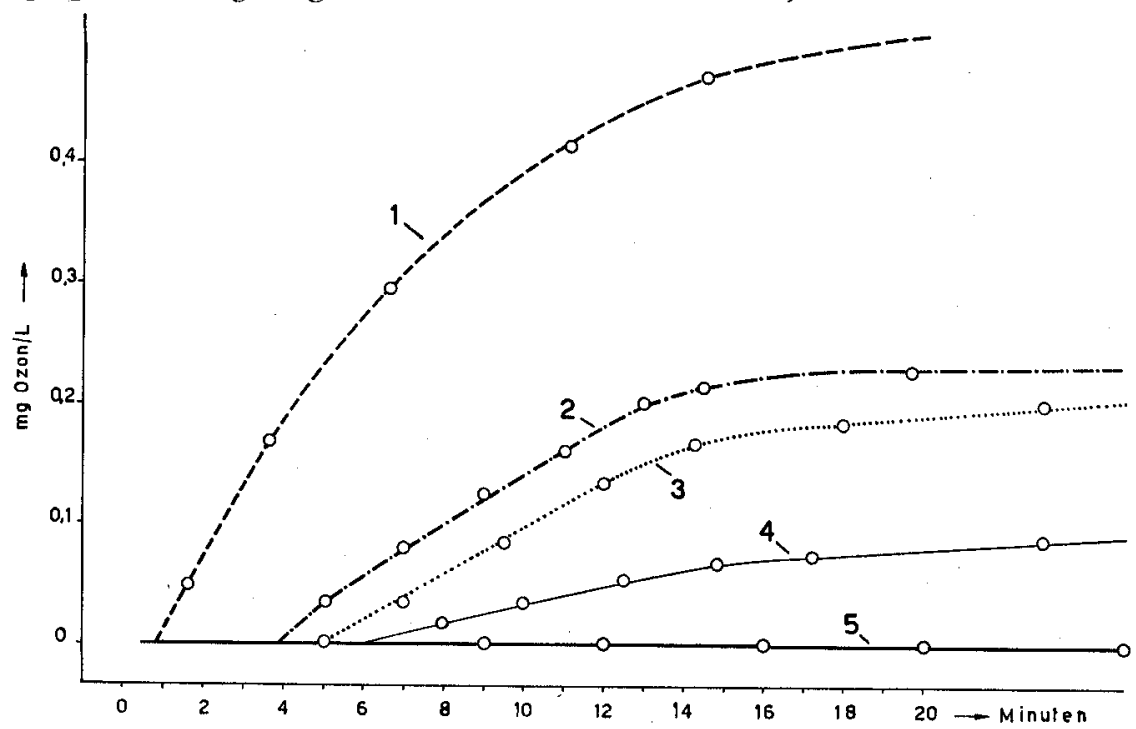

Figur 1. Die Veränderung der Ozonkonzentration während der Ozonisierung: Ozonleistung $=1,57 \mathrm{mg} \mathrm{O} / \mathrm{min}$. Die Durchmischungsbedingungen wurden konstant gehalten. 1. Quellwasser Zürich (Sihl-Lorze) ; 2. Zürichseewasser, filtriert; 3. Zürichseewasser, vorfiltriert; 4. Zürichseewasser, unfi]triert; 5. Mischung aus destilliertem Wasser und Abwasser im Verhältnis 10:1.

gewisse Charakterisierung des Wassers ermöglicht wird. Zudem geben solche Versuche Aufschluss über das Ozonzehrungsvermögen der betreffenden Wässer und vermitteln Anhaltspunkte für die Dimensionierungsgrundlagen technischer Ozonisierungsanlagen. Einige Beispiele solcher Kurven sind in Figur I dargestellt.

In unseren Versuchen konnten wir drei verschiedene Wassertypen unterscheiden (vgl. schematische Darstellung in Fig. 2). 


\section{Typus A (zum Beispiel: Bidestilliertes Wasser)}

Die Kurve für bidestilliertes Wasser entspricht einer Gasabsorptionskurve. Die Ozonabsorption erfolgt nach einer Reaktion erster Ordnung. Ihre Geschwindigkeit ist abhängig vom Löslichkeitskoeffizienten bei der entsprechenden Temperatur und vom Wirkungsgrad der verwendeten Vermischungseinrichtung.

Wir haben für solches ozonzehrungsfreies Wasser den Löslicbkeitskoeffizienten

$$
q=\frac{\mathrm{O}_{3} w}{\mathrm{O}_{3} g}
$$

bestimmt, wobei $\mathrm{O}_{3 w}$ die Konzentration von Ozon in der wässerigen, $\mathrm{O}_{3 g}$ die Ozonkonzentration in der Gasphase bedeuten. Unsere Bestimmungen wurden bei einem Barometerstand von $710-730 \mathrm{~mm}$ durchgeführt. Die in Tabelle I zusammengestellten Werte sind nicht auf Normalzustand korrigiert. Da der Salzgehalt des Wassers die Löslichkeit beeinflusst, haben wir alle Versuche bei einer ionalen Stärke von $\boldsymbol{\mu}=5 \cdot \mathrm{IO}^{-2}$ (bidestilliertes Wasser $+\mathrm{Na}_{2} \mathrm{SO}_{4}$ und/oder $\mathrm{NaHCO}_{3}, \mathrm{Na}_{2} \mathrm{CO}_{3}$, Kohlensäure) durchgeführt. Die Ozonlöslichkeit ist bis hinauf zu pH-Werten von 8,5 pH-unabhängig. Bei höheren pH-Werten sinkt die Löslichkeit.

Tabelle 1

Löslicbkeitskoeffizient fïr Ozon in Wasser bei $\mu=5 \cdot 10^{-2}$

(Interpolationswerte, die aus Löslichkeitsbestimmungen bei verschiedenen Temperaturen und bei einem Barometerstand von $710-730 \mathrm{~mm}$ erhalten wurden. Die Werte sind nicht auf Normalzustand korrigiert)

\begin{tabular}{lcccccccc}
\hline Temperatur ${ }^{\circ} \mathrm{C}$ & 5 & 8 & 10 & 13 & 15 & 17 & 20 & 25 \\
Löslichkeitskoeffizient & 0,43 & 0,41 & 0,39 & 0,375 & 0,35 & 0,33 & 0,32 & 0,28 \\
\hline
\end{tabular}

\section{Typus B (zum Beispiel: Grundwasser)}

Beim Ozonisieren von Grundwasser erhält man in der Regel bei der Auftragung der zeitlichen Veränderung der Ozonkonzentrationen eine analoge Kurve wie bei einem ozonzehrungsfreien Wasser, allerdings mit dem Unterschied, dass der Anstieg der Kurve erst nach einer gewissen Zeitspanne (vgl. Fig. 2) beginnt.

Unsere Versuche haben gezeigt, dass die Ozonmenge, die in der Zeitspanne 0 bis $t$ verbraucht wird, einigermassen konstant ist. Für jede Ozonleistung kann somit bei gegebener Vermischungseinrichtung die minimale erforderliche Kontaktzeit zwischen Ozon und Wasser berechnet werden, die notwendig ist, um das Auftreten von «freiem Ozon» im 
Wasser zu bewirken. Kurven vom Typus $B$ haben wir für alle von uns untersuchten Grundwässer erhalten. Wir vermögen jedoch nicht auszusagen, welche Substanzen im Grundwasser den Ozonverbrauch bewirken. Die für Grundwässer meist ohnehin niedrige Permanganatzahl wird durch Ozonisierung des Wassers wenig beeinflusst.

\section{Typus C (zum Beispiel: Oberflächenmasser)}

Ein Wasser vom Typus $C$ ergibt beim Ozonisieren eine Ozon-Zeitkurve, die erheblich flacher verläuft als die entsprechende Kurve eines ozon-

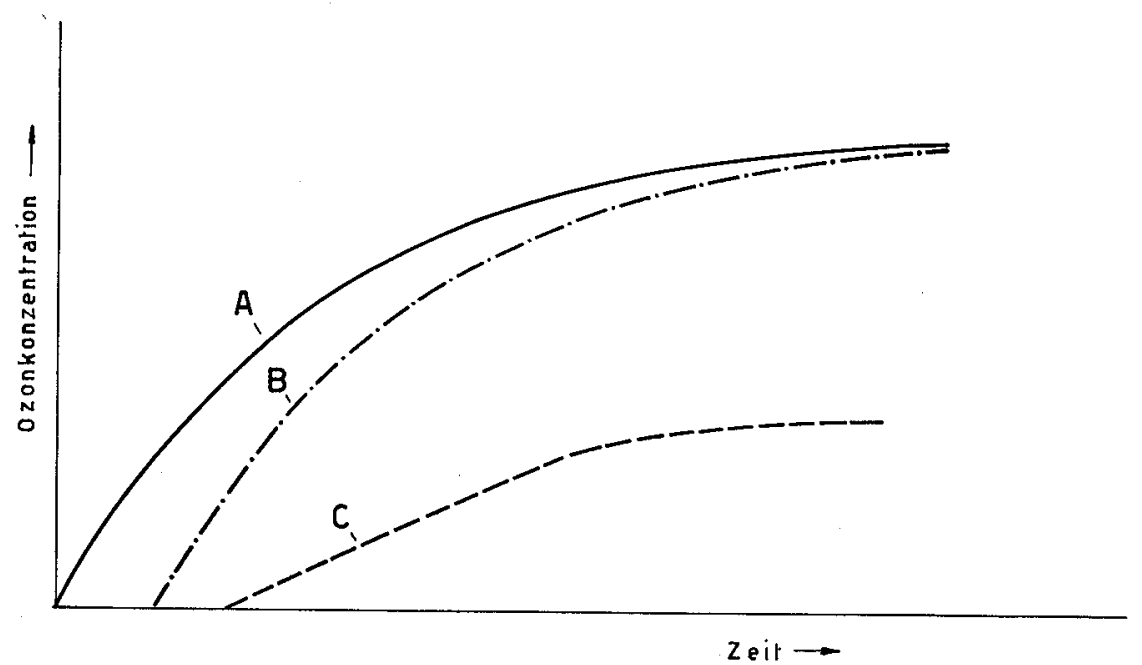

Figur 2. Schematische Darstellung der Veränderung der Ozonkonzentration während des Ozonisierens der drei beschriebenen Wassertypen. Typus A: ozonzehrungsfreies Wasser; Typus B: zum Beispiel Grundwasser; Typus C: zum Beispiel Oberflächenwasser.

zehrungsfreien Wassers und eines Wassers vom Typus B. Beim Wasser vom Typus $C$ wird auch bei stundenlangem Ozonisieren der Gleichgewichtswert der Ozonkonzentration, der durch den Löslichkeitskoeffizienten gegeben ist, nicht erreicht (vgl. Figuren). Solche Kurven haben wir für alle bis jetzt untersuchten Oberflächenwässer sowie für Mischungen von destilliertem Wasser mit Spuren von Abwasser erhalten. Während bei einem Wasser vom Typus $B$ offenbar nur solche Substanzen vorhanden sind, die in einfacher Reaktion eine Ozonzehrung bewirken, muss man annehmen, dass Wässer vom Typus $C$ solche organische Verbindungen enthalten (z. B. Enzyme), die den Zerfall von Ozon katalytisch beeinflussen.

Sobald die Zerfallsgeschwindigkeit Werte erreicht, die sich grössenordnungsmässig der Absorptionsgeschwindigkeit von Ozon im Wasser 
nähern, muss ein langsamerer Anstieg der Ozonkonzentration stattfinden. Es ist dies eine Erscheinung, die auch beim Ozonisieren eines alkalischen Wassers ( $\mathrm{pH}=9$ und darüber) beobachtet werden kann. Wie wir durch kinetische Untersuchungen eindeutig nachweisen konnten [2], katalysieren Hydroxylionen den Ozonzerfall in stärkstem Masse. Wir wissen jedoch nicht, von welchen Substanzen in Oberflächenwässern der Ozonzerfall so stark begünstigt wird.

Die auffälligen Unterschiede im Ozonzehrungsvermögen von Grundwässern und Oberflächenwässern lassen sich zur Charakterisierung der beiden Typen beiziehen. So sollte es zum Beispiel auch möglich sein, in besonderen Fällen eine Infiltration von Oberflächenwasser ins Grundwasser nachweisen zu können.

\section{ZUSAMMENFASSUNG}

I. Wässerige Ozonlösungen weisen einen komplizierteren Chemismus auf, als es den Anschein machen könnte. Als bakterizide Agentien sind nicht nur Ozon, sondern auch verschiedene Ozonzerfalls- und -oxydationsprodukte in Betracht zu ziehen.

2. Eine spezifische Methode zur analytischen Bestimmung des molekularen Ozons, wie sie von ZEHENDER und STUMM [6] beschrieben wurde, ist von grösster Wichtigkeit in der Untersuchung der Wasserozonisierung, insbesondere auch zur sinnvollen Interpretation der Wirksamkeit von Ozon.

3. Grundwässer und Oberfächenwässer unterscheiden sich in auffallender Weise in ihrem Verhalten gegenüber Ozon. Die beiden Wassertypen zeigen charakteristische Ozonabsorptionskurven. Spuren von gewissen Substanzen genügen, um den Zerfall des Ozons in wässeriger Lösung stark zu beschleunigen.

\section{LITERATURVERZEICHNIS}

[I] Fatr, G. M., Morris,J.C., Chang, S. L., Ira Weil und Burden, R. P., J.Amer. Water Works Ass. 40, IO5I (1948).

[2] Stumm, W., Helv. chim. Acta 37, 773 (r954).

[3] Aldder, M. G., und Hill, G. R., J. Amer. chem. Soc. 72, I884 (1950).

[4] WeIss, J., Trans. Faraday Soc. 31, 668 (1935).

[5] Wuhrmann, K., und Meyrath, J., Schw. Z. Path. Bakt. 18, I060 (1955).

[6] Zehender, F., und Stumm, W., Mitt. Lebensm. Hyg. 44, 206 (I953).

[7] Latimer, W. M., Oxidation Potentials (Prentice Hall, New York 1953).

[8] RIEcHE, A., Alkylperoxyde und Ozonide (Steinkopff Dresden 1936). 